\title{
Haushaltsüberschüsse, konservative Parteien und das Trilemma der Fiskalpolitik
}

\author{
Lukas Haffert / Philip Mehrtens
}

\section{Budget Surpluses, Conservative Parties, and the Trilemma of Fiscal Policy}

Abstract: This paper analyzes the fiscal strategies of conservative parties in countries running sustained budget surpluses. It argues that conservatives can employ a hard balanced budget norm to skirt resistance against public spending cuts and to further their goal of restricting the size of the state. Whereas the "starving the beast" approach to shrinking the state failed to enforce spending cuts, budget surpluses did create the fiscal and political conditions for tax cuts. This argument is theoretically explored with the concept of a trilemma of fiscal policy. Afterwards, two case studies of Sweden and Australia analyze the partisan politics of ongoing retrenchment in surplus times.

Keywords: Budget surplus, fiscal policy, conservative parties, Australia, Sweden

Schlagwörter: Haushaltsüberschüsse, Fiskalpolitik, konservative Parteien, Australien, Schweden

\section{Einleitung}

„This deficit is no despised orphan. It is President Reagan's child, and secretly he loves it." The New York Times, 25. Januar 1987

Mit diesen Worten kommentierte die New York Times Ronald Reagans Rede zur Lage der Nation im Januar 1987. Sie bezog sich damit auf eine fiskalpolitische Strategie, die unter dem Titel „Starving the Beast“ bekannt geworden ist. Demnach können konservative Parteien Defizite strategisch einsetzen, um Ausgabenkürzungen durchzusetzen, die ohne den Druck des Defizits nicht möglich wären.

Die These, dass der Budgetsaldo als strategisches Instrument im Parteienwettbewerb eingesetzt werden kann, stieß in der Literatur zur strategischen Verschuldung (Persson u. Svensson 1989; Alesina u. Tabellini 1990) auf ein breites Echo. Demnach strebt eine Partei einen bestimmten Haushaltssaldo nicht deshalb an, weil sie diesen Saldo selbst für wünschenswerte Politik hält, sondern weil sie damit rechnet, dass der Saldo politisch attraktive Folgewirkungen auslösen wird. Der Saldo wird damit von einem Ziel zu einem Mittel der Politik.

In Übereinstimmung mit der These von „Starving the Beast“ ist dieses Argument bislang stets auf den strategischen Einsatz von Haushaltsdefiziten angewendet worden. In dieser Studie wird dagegen der umgekehrte Fall analysiert. Demnach können nicht nur Haushaltsdefizite, sondern auch Haushaltsüberschüsse im Parteienwettbewerb strategisch eingesetzt werden. Säkular-konservative Parteien, 
die eine geringere Rolle des Staates in der Wirtschaft anstreben, können die Logik von „Starving the Beast" umkehren. ${ }^{1}$ In einer Strategie, die als „Overfeeding the Beast" bezeichnet werden kann, dienen ausgeglichene Haushalte ihnen als Instrument, um einen dauerhaften Rückbau des Staates zu erwirken. Die Bewahrung des Überschusses rechtfertigt dann eine Politik fortgesetzter Kürzungen der Staatsausgaben bei gleichzeitigen Steuersenkungen.

Die Studie gliedert sich wie folgt: Zunächst entwickeln wir ein einfaches theoretisches Argument über die Rolle einer Haushaltsausgleichsnorm im Parteienwettbewerb. Dabei legen wir ein besonderes Augenmerk auf die verschiedenen strategischen Optionen konservativer Parteien. Anschließend diskutieren wir das Universum der Fälle, in denen diese Norm empirisch wirksam wurde, also Länder, die für einen längeren Zeitraum ausgeglichene Haushalte oder sogar Überschüsse erzielten. Dabei zeigen wir mit einem einfachen quantitativen Vergleich, dass die Entwicklung der Staatstätigkeit in diesen Ländern tatsächlich stark mit den Präferenzen konservativer Parteien übereinstimmte. Auf Basis dieser Diskussion wird dann in zwei Fallstudien die Überschusspolitik Schwedens und Australiens detaillierter analysiert, um die Rolle der strategischen Positionierung konservativer Parteien im Parteienwettbewerb herauszuarbeiten.

\section{Konservative Parteien und das Trilemma der Fiskalpolitik}

Der Grundgedanke unseres Arguments ist, dass konservative Parteien ein Bekenntnis zu ausgeglichenen Haushalten als strategisches Instrument einsetzen können, um auch andere konservative Ziele in der Fiskalpolitik zu erreichen. Um dieses Argument genauer zu entwickeln, ist ein theoretischer Rahmen nötig, mit dessen Hilfe verschiedene fiskalpolitische Strategien und die Rolle ausgeglichener Haushalte in diesen Strategien analysiert werden können. Zu diesem Zweck wird im Folgenden das Konzept eines Trilemmas der Fiskalpolitik entwickelt (Abbildung 1).

Dieses Trilemma beschreibt die strategische Ausgangssituation, vor der konservative Parteien bei der Wahl ihrer fiskalpolitischen Strategie stehen. Es ergibt sich aus der Tatsache, dass der Budgetsaldo die Differenz aus Staatseinnahmen und Staatsausgaben ist. Dieser Zusammenhang beschreibt aber nicht nur eine arithmetische Gesetzmäßigkeit, sondern auch einen strategischen Zielkonflikt. Er konfrontiert politische Parteien mit drei fiskalpolitischen Zielen - höhere Staatsausgaben, niedrigere Steuern und ausgeglichene Haushalte - von denen sich immer nur zwei gleichzeitig verwirklichen lassen. ${ }^{2}$ Alle drei Ziele sollten bei den Wählern auch auf prinzipielle Zustimmung stoßen. Weil aber immer nur zwei dieser Ziele

1 Das im Folgenden entwickelte Argument bezieht sich explizit auf die Strategien solcher säkularkonservativen Parteien. Diese treten in den skandinavischen und den angelsächsischen Ländern traditionell für einen schlanken Staat ein (Kitschelt 2001, S. 281). Dagegen stehen die christdemokratischen Parteien Mitteleuropas einem starken Staat deutlich positiver gegenüber (Kalyvas u. van Kersbergen 2010).

2 Es ließe sich einwenden, Befürworter eines schlanken Staates stünden hier vor gar keinem Zielkonflikt. Auch konservative Parteien haben jedoch in aller Regel starke Ausgabenpräferenzen in bestimmten Politikfeldern, z. B. der inneren Sicherheit oder der Verteidigung. Außerdem haben starke Kürzungen häufig negative Auswirkungen auf das Wahlergebnis. 
zugleich erreicht werden können, sind die Parteien gezwungen, eines den anderen beiden unterzuordnen. Bildlich gesprochen besetzen sie mit ihrem politischen Programm eine Ecke des in Abbildung 1 skizzierten Dreiecks. Eine solche Prioritätensetzung wird im Folgenden als die „fiskalische Strategie“ einer Partei bezeichnet.

\section{Abbildung 1: Das strategische Trilemma der Fiskalpolitik}

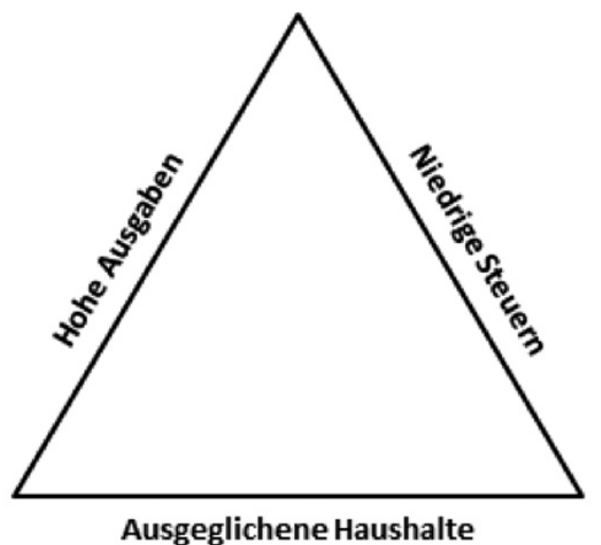

Quelle: Eigene Darstellung.

Bei den drei möglichen fiskalischen Strategien handelt es sich um abstrakte Idealtypen, und die reale Politik stellt stets einen Kompromiss zwischen ihnen dar. Dennoch sind die mit ihnen verbundenen Prioritätensetzungen bei unterschiedlichen konservativen Regierungen zu verschiedenen Zeitpunkten klar erkennbar. Der klassische Fiskalkonservatismus, wie er zum Beispiel von der republikanischen Eisenhower-Administration in den USA vertreten wurde, setzte seine Priorität auf ausgeglichene Haushalte und war bereit, die dafür nötigen Steuern zu erheben, wenn keine Ausgabenkürzungen möglich waren (Stein 1969). Der von Ronald Reagan repräsentierte Angebotskonservatismus der 1980er-Jahre gab das Ziel eines ausgeglichenen Haushalts dagegen zugunsten von Steuersenkungen und einer Erhöhung des Verteidigungsetats auf. Im Dreieck von Abbildung 1 entspricht dies einer Verschiebung der fiskalischen Strategie von unten links nach oben. Die dritte mögliche fiskalische Strategie vereint einen ausgeglichenen Haushalt mit niedrigeren Steuern und reduziert im Gegenzug die öffentlichen Ausgaben. Sie wird durch die rechte untere Ecke des Dreiecks in Abbildung 1 symbolisiert und soll durch „Starving the Beast“ erreicht werden.

Konservative Parteien haben ihre fiskalischen Strategien also wiederholt geändert. Solche Strategiewechsel haben sich auch in der theoretischen Konzeptualisierung des Parteieneinflusses in der Fiskalpolitik niedergeschlagen. ${ }^{3}$ Dabei nahm die

3 Da es hier um die spezifische Frage strategischen Verhaltens geht, verzichten wir auf eine allgemeine Darstellung der überaus umfangreichen Literatur zu Parteiendifferenzen in der Fiskalpolitik (vergleiche aber die Übersichtsdarstellungen in Boix 2000; Franzese 2002). 
erste Welle von politikwissenschaftlichen und ökonomischen Untersuchungen im Gefolge von Hibbs (1977) und Tufte (1978) an, linke Parteien verschuldeten sich stärker als rechte Parteien. Die Jahre der Reagan-Regierung stellten diese These infrage (siehe auch die empirischen Befunde in Wagschal 1996; Cusack 1999). Dieser konservative Strategiewechsel inspirierte die Entstehung der Literatur zur sogenannten „strategischen Verschuldung“ (Persson u. Svensson 1989; Alesina u. Tabellini 1990). Demnach kann eine Regierung, die davon ausgeht, abgewählt zu werden, sich bewusst verschulden, um den Entscheidungsspielraum der zukünftigen Regierung einzuschränken. Damit kann die neue Regierung an Reformen gehindert und die eigene Politik langfristig festgeschrieben werden. Ausgerechnet besonders hartnäckige („stubborn“) Konservative könnten daher zu Defiziten greifen, um progressive Nachfolgeregierungen zu Einsparungen zu zwingen und von neuen Staatsausgaben abzuhalten.

Dass ein solches strategisches Verhalten der Regierung prinzipiell denkbar ist, ist auch eine Implikation einiger wichtiger Ergebnisse der jüngeren Literatur. Diese betont, dass Parteien nicht mechanisch die Präferenzen der von ihnen vertretenen sozialen Gruppen zum Ausdruck bringen. Vielmehr verfügt die Parteiführung über eine gewisse Unabhängigkeit von Parteimitgliedern und Wählern und kann diese nutzen, um sich strategisch zu verhalten (Häusermann et al. 2013). Insbesondere berücksichtigen Parteien bei der Wahl ihrer Positionen die Standpunkte ihrer Konkurrenten. Beispielsweise versuchen Oppositionsparteien in wirtschaftlich guten Zeiten regelmäßig, die politische Distanz zur Regierung zu minimieren, um diese daran zu hindern, ihre Erfolge politisch zu nutzen (Hellwig 2012). Auch zeigen diese Arbeiten, dass Parteien auf Positionswechsel ihrer Konkurrenten reagieren, indem sie ihre eigene politische Position anpassen (Adams u. Somer-Topcu 2009). Dabei spielt die allgemeine strategische Konfiguration des Parteienwettbewerbs eine wichtige Rolle: Beispielsweise haben Parteien der linken Mitte sehr viel geringere strategische Spielräume, wenn sie mit einer kleineren linken Partei konkurrieren, als wenn dies nicht der Fall ist (Kitschelt 2001; Picot 2009).

Fiskalpolitische Strategiewechsel sind demnach nicht allein Ausdruck politischer Präferenzverschiebungen. Vielmehr sind die Parteien in der Wahl ihrer fiskalischen Strategie von zwei Randbedingungen beschränkt: Zum einen müssen sie auf die allgemeinen fiskalpolitischen Umstände reagieren, die sie dazu zwingen, bestimmte Positionen einzunehmen. Zum anderen ist der Erfolg jeder Strategie von der strategischen Positionierung der Konkurrenten abhängig. Die Wahl einer fiskalischen Strategie beeinflusst einerseits das Verhalten der anderen Parteien, hängt aber andererseits auch von deren Verhalten ab.

Die allgemeinen fiskalischen Umstände können mit Pierson (2001) als das herrschende „fiskalische Regime“ bezeichnet werden. Fiskalpolitische Entscheidungen werden demnach in einem relativ stabilen Makrokontext getroffen, an den sich alle Parteien anpassen müssen. Pierson argumentiert: „The dynamics of taxing and spending are shaped and reinforced by a particular political context of institutions, powerful organizations, public policies and dominant ideas" (Pierson 2001, S. 57). Bezüglich des Trilemmas bedeutet dies, dass das herrschende Regime einen starken Einfluss darauf hat, zu welchem fiskalpolitischen Ziel sich beide Parteien bekennen und welche beiden Ziele zwischen ihnen umstritten sind. 
Empirisch unterscheidet Pierson zwei seit 1945 herrschende fiskalische Regime. Bis Mitte der 1970er-Jahre existierte demnach ein „expansives Regime“, das auf einen Ausbau der Rolle des Staates gerichtet war: Hohe Wachstumsraten, die Friedensdividende, starke Gewerkschaften, geringer Steuerwiderstand und das vorteilhafte demografische Profil eines sich entwickelnden Wohlfahrtsstaates bewirkten, dass sich praktisch alle Parteien zu einer Ausweitung der Staatstätigkeit bekannten.

In den 1970er-Jahren kam es zu einem folgenreichen Regimewechsel: Das expansive Regime wurde von einem „Austeritätsregime“ abgelöst, dass die Fiskalpolitik noch heute prägt. Praktisch alle Faktoren, die das expansive Regime stützten, wirken seitdem in die entgegengesetzte Richtung (Pierson 2001, S. 61-68.): Die Wachstumsraten gingen dauerhaft zurück, der ausgereifte Wohlfahrtsstaat litt unter einem immer ungünstigeren demografischen Profil, die Friedensdividende der Nachkriegszeit war längst verzehrt, die Gewerkschaften verloren an Einfluss und der Steuerwiderstand in der Bevölkerung wuchs. Für das Austeritätsregime sind daher ein permanenter Druck auf die Staatsausgaben und immer größere Schwierigkeiten bei der Generierung von Staatseinnahmen charakteristisch.

Der strategische Charakter des Umgangs einer Regierung mit diesen Rahmenbedingungen entsteht aber erst, weil sie die mögliche Reaktion der Opposition berücksichtigen muss. Die fiskalische Strategie der Regierung kann defensiv dazu dienen, eine bestimmte Reaktion der Opposition zu verhindern. Sie kann aber auch offensiv eingesetzt werden, um eine bestimmte Positionierung der Opposition zu erzwingen. Wenn die Regierung etwa für ausgeglichene Haushalte und niedrigere Steuern eintritt, könnte die Opposition die Strategie der Regierung kopieren und diese Ziele ebenfalls bekräftigen. Sie könnte sich aber auch für höhere Staatsausgaben aussprechen. In diesem Fall hat sie zwei Möglichkeiten: Einerseits kann sie zu deren Finanzierung höhere Steuern verlangen, andererseits für eine Defizitfinanzierung eintreten. Je nachdem, für welche Strategie sie sich entscheidet, ergibt sich eine unterschiedliche fiskalpolitische Konfliktlinie. Da die Regierung aber um die wahrscheinliche Positionierung der Opposition weiß, wird sie diese bereits bei der Formulierung ihrer eigenen Strategie berücksichtigen. Dabei wird sie zudem kalkulieren, wie sicher oder unsicher ihre eigene politische Position ist und wie glaubwürdig die Opposition eine bestimmte Position vertreten kann (Hicks u. Swank 1992; Kitschelt 2001; Franzese 2002). Wenn die Opposition beispielsweise selbst hohe Defizite zu verantworten hatte, wird es ihr schwerfallen, ausgeglichene Haushalte zu politisieren.

Im Kontext des strategischen Trilemma-Modells versucht „Starving the Beast“, den politischen Gegner durch umfangreiche Steuersenkungen dazu zu zwingen, die Verteidigung hoher Staatsausgaben aufzugeben. ${ }^{4}$ Das Instrument, das die Opposition zu diesem Kurswechsel bewegen soll, ist das wachsende Defizit. Wenn die Opposition ausgeglichenen Haushalten Priorität vor hohen Staatsausgaben ein-

4 Dabei ist es für das hier entwickelte Argument weitgehend unerheblich, ob es sich bei „Starving the Beast" tatsächlich um eine bewusst eingesetzte Strategie oder um eine ex-Post-Rationalisierung handelt. Letztere Position wurde besonders von Reagans Budgetdirektor David Stockman (1986) vertreten, während Bartlett (2007) die Wurzeln von „Starving the Beast“ im konservativen Gedankengut der 1970er-Jahre zeigt. Solange Defizite tatsächlich zu den beschriebenen Effekten führen, besteht jedenfalls die Möglichkeit, sie strategisch einzusetzen. 
räumt, wird sie zu einem Kompromiss bereit sein, der einen Haushaltsausgleich bei nun gesunkenen Steuern und Staatsausgaben erlaubt und damit die eigentlichen konservativen Politikpräferenzen verwirklicht.

Empirisch blieben die erwarteten Ausgabensenkungen in den USA jedoch aus. In einer umfangreichen Untersuchung finden Romer und Romer (2009, S.1) „no support for the hypothesis that tax cuts restrain government spending." Stattdessen führten Reagans Steuersenkungen zu Defiziten in bis dahin nicht gekanntem Ausmaß. Selbst Befürworter eines schlanken Staates betrachten „Starving the Beast" daher heute als Fehlschlag (Niskanen 2006). Stattdessen hat in den letzten Jahren eine umgekehrte Lesart, in der Steuererhöhungen zu wachsenden Staatsausgaben führen, an Popularität gewonnen (Bartlett 2007).

Wir argumentieren, dass der Grund für das Scheitern von „Starving the Beast“ im Fehlen einer harten Budgetausgleichsnorm lag. Da Kürzungen der öffentlichen Ausgaben hochgradig unpopulär waren, gelang es nicht, die erwarteten Ausgabenkürzungen zu erzwingen (Morgan 2009). Ohne die Existenz einer Budgetausgleichsnorm bestand für linke Parteien kein Anlass, einer Kürzung der Staatsausgaben zuzustimmen, vielmehr war es politisch viel attraktiver, diese besonders entschieden zu verteidigen.

Die Institutionalisierung einer strikten Budgetausgleichsnorm bietet konservativen Parteien dagegen die Möglichkeit, den Widerstand gegen Kürzungen zu umgehen. Die politische Verpflichtung, das Budget im Überschuss zu halten, hilft dabei, die gewünschten Ausgabenkürzungen durchzusetzen und schafft zudem den fiskalischen Spielraum für Steuersenkungen. In diesem Fall sind nicht Steuersenkungen das strategische Instrument, um Ausgabenkürzungen zu erzwingen, sondern ausgeglichene Haushalte das Mittel, um Steuersenkungen zu ermöglichen. Im Gegensatz zu „Starving the Beast“ lässt sich diese Strategie als „Overfeeding the Beast" bezeichnen (Abbildung 2).

Abbildung 2: Konservative Strategien zur Reduktion der Staatstätigkeit: „Starving the Beast" und Überschusspolitik

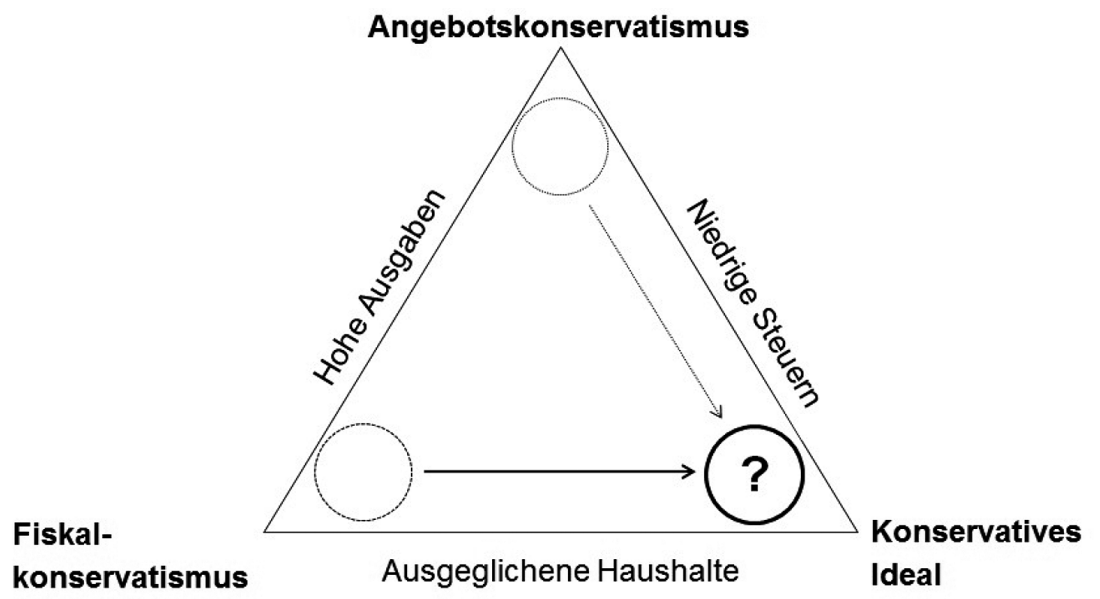

Quelle: Eigene Darstellung. 
Während die „Starving the Beast“-Strategie eine Bewegung entlang der Steuersenkungs-Kante des Trilemmas anstrebt, zielt die „Overfeeding the Beast“-Strategie auf eine Bewegung entlang der Haushaltsausgleichs-Kante ab. Dabei stellt sie linke Parteien vor das Dilemma, zur Finanzierung höherer Staatsausgaben entweder unpopuläre Steuererhöhungen vorschlagen oder den Überschuss infrage stellen zu müssen. Häufig wird es als Folge wahltaktisch und politisch unattraktiv, den fiskalpolitischen Kurs der Konservativen grundlegend zu kritisieren, und linke Parteien befürworten eine strikte Haushaltsausgleichsnorm ebenfalls (,issue convergence" [Arndt 2014]).

Bei der Analyse dieser Strategie stehen nicht mehr die politischen Folgewirkungen von Haushaltsdefiziten, sondern von Haushaltsüberschüssen im Mittelpunkt. Ihre Voraussetzung ist, dass es konservativen Parteien gelingt, tatsächlich einen von allen relevanten Parteien geteilten Konsens über die Notwendigkeit ausgeglichener Haushalte herbeizuführen und für die eigenen Zwecke nutzbar zu machen. Die Frage, wie dies gelingt, steht im Zentrum der beiden folgenden Fallstudien. Zuvor wird jedoch kurz im Ländervergleich gezeigt, dass diese Politik tatsächlich zu den von den Konservativen gewünschten Ergebnissen führte.

\section{Haushaltsüberschüsse und Staatstätigkeit im internationalen Vergleich}

Auf Basis einer intuitiven Empirie, die von dem in der Literatur vielfach diagnostizierten „Deficit Bias“ repräsentativer Demokratien ausgeht, wirkt es wenig erfolgversprechend, eine explizite politische Haushaltsausgleichsnorm zum Ausgangspunkt einer theoretischen und empirischen Untersuchung $\mathrm{zu}$ machen. Tatsächlich ist das empirische Bild jedoch wesentlich uneinheitlicher. Selbst wenn man Norwegen ausschließt, wiesen die „klassischen“ OECD-Länder zwischen 1980 und 2009 in mehr als einem Fünftel aller Jahre einen Haushaltsüberschuss auf. Diese Überschüsse verteilen sich jedoch sehr unausgewogen auf die einzelnen Länder (Tabelle 1). Vier Länder erzielten überhaupt keine, neun Länder nur vereinzelte Überschüsse und nie für längere Zeiträume. Damit entfielen mehr als zwei Drittel der Haushaltsüberschüsse auf nur sieben Länder - Australien, Dänemark, Finnland, Irland, Kanada, Neuseeland und Schweden.

Tabelle 1: Haushaltsüberschüsse in OECD-Ländern, 1980-2009

\begin{tabular}{l|l} 
Land & Haushaltsüberschuss \\
\hline Australien & $1998-2007$ \\
Belgien & $2001 ; 2006$ \\
Dänemark & $1986-1989 ; 1999-2008$ \\
Deutschland & $1989 ; 2000 ; 2007-2008$ \\
Finnland & $1980-1990 ; 1998-2008$ \\
Frankreich & $/$ \\
Großbritannien & $1999-2001$ \\
Irland & $1997-2007$ \\
Island & $1999-2000 ; 2004-2007$ \\
Italien & $/$
\end{tabular}




\begin{tabular}{l|l} 
Land & Haushaltsüberschuss \\
\hline Japan & $1988-1992$ \\
Kanada & $1997-2007$ \\
Neuseeland & $1994-2008$ \\
Niederlande & $1999-2000 ; 2006-2008$ \\
Österreich & $/$ \\
Portugal & $/$ \\
Schweden & $1987-1990 ; 1998-2008$ \\
Schweiz & 2006-offen \\
Spanien & 2005-2007 \\
USA & $1998-2000$
\end{tabular}

Quelle: OECD Economic Outlook Database, Europäische Kommission.

Diese sieben Länder stehen im Mittelpunkt der folgenden Untersuchung, wobei Irland von der Untersuchung ausgeschlossen wird. Der irische Überschuss war vor allem Ausdruck einer Immobilienblase sowie einer allgemeinen Überhitzung der Wirtschaft und ist insofern kaum für einen Vergleich geeignet. Damit verbleiben sechs Länder, die in Tabelle 2 vorgestellt werden. Sie alle erzielten seit Mitte der 1990er-Jahre für mehr als eine Dekade praktisch ununterbrochen Haushaltsüberschüsse und bauten ihre Nettoverschuldung fast vollständig ab.

Tabelle 2: Fiskalische Indikatoren in sechs Überschussländern, in Prozent des BIP

\begin{tabular}{l|cccccc} 
Land & $\begin{array}{c}\text { Überschuss } \\
\text { seit }\end{array}$ & $\begin{array}{c}\varnothing \text { Über- } \\
\text { schuss }\end{array}$ & \multicolumn{2}{c}{ Bruttoverschuldung } & \multicolumn{2}{c}{ Nettoverschuldung } \\
& 1998 & 0,86 & 37,0 & 14,2 & 21,2 & $-7,3$ \\
\hline Australien & 1999 & 2,39 & 72,4 & 34,3 & 35,1 & $-3,8$ \\
Dänemark & 19907 & in $\boldsymbol{t}$-1 & in 2007 \\
Finnland & 1998 & $3,48^{5}$ & 64,8 & 41,4 & $-7,5$ & $-72,6$ \\
Kanada & 1997 & 0,98 & 101,7 & 66,5 & 68,5 & 22,4 \\
Neuseeland & 1994 & 2,68 & 63,8 & 25,7 & 50,5 & $-5,5$ \\
Schweden & 1998 & 1,23 & 83,0 & 49,0 & 24,6 & $-22,4$ \\
Durchschnitt & $/$ & 1,94 & 70,5 & 38,5 & 32,1 & $-14,9$
\end{tabular}

Quelle: OECD Economic Outlook Database, eigene Berechnungen.

In diesen sechs Ländern bildete sich offenbar eine politische Haushaltsausgleichsnorm heraus, die durch entsprechende institutionelle Reformen, eine Neukonfiguration der politischen Interessen und eine ideelle Tabuisierung von Defiziten stabilisiert wurde. ${ }^{6}$ Diese Norm beeinflusste auch den Parteienwettbewerb. So wurden

5 Finnlands extrem hohe Überschüsse erklären sich aus einer Besonderheit seines Rentensystems, das eine kapitalgedeckte Säule enthält, die über das staatliche Versicherungssystem organisiert wird und jährliche Rücklagen bildet, die in den 1990er-Jahren zeitweilig mehr als drei Prozent des BIP erreichten. Die OECD rechnet diese Rücklagen dem Budgetsaldo des Gesamtstaates zu.

6 Vgl. dazu ausführlich: Haffert (2014). 
alle Regierungen, die einen dauerhaften Überschuss erzielten, bei der ersten Wahl im Überschuss wiedergewählt. Dies bestätigt Brenders und Drazens (2008) Resultat, dass die Wiederwahlchancen einer Regierung positiv mit dem Haushaltssaldo korrelieren.

Allerdings nimmt die Bedeutung von Parteiendifferenz im Überschuss ab, da die „Overfeeding the Beast"-Strategie auf der Herbeiführung eines Allparteienkonsenses über die Haushaltsausgleichsnorm basiert. Der Einfluss von Parteien besteht vielmehr in ihrer strategischen Interaktion innerhalb des Trilemmas der Fiskalpolitik. Diese Interaktion und ihre Auswirkungen sollen im Folgenden analysiert werden. Die Tatsache, dass es nur sechs Länder mit dauerhaften Überschüssen gibt, macht es jedoch schwierig, die „Overfeeding the Beast“-Strategie ökonometrisch zu testen. Vor diesem Hintergrund beschränken wir uns im Folgenden auf die deskriptive Analyse der Entwicklung der aggregierten Staatsaugaben und -einnahmen und analysieren die Dynamik des Parteienwettbewerbs in zwei Fallstudien.

Konkret vergleicht Tabelle 3 die Länder, die in den späten 1990er-Jahren eine Überschussnorm institutionalisierten, zum einen mit den vier Ländern, die parallel für einige wenige Jahre Überschüsse erwirtschafteten und zum anderen mit den neun Ländern, die trotz des Konjunkturaufschwungs dieser Zeit durchgängig im Defizit verblieben. ${ }^{7}$ Sie zeigt die Veränderung der zyklisch bereinigten Staatseinnahmen bzw. Staatsausgaben zwischen 1990, 1998 und 2007. Das Jahr 2007 war das letzte Jahr vor der Weltfinanzkrise, 1998 der Höhepunkt der weltweiten Konsolidierungswelle der späten 1990er-Jahre und 1990 der Beginn einer weltweiten Rezession, die mit einem enormen Anstieg der Schuldenquoten verbunden war.

Dabei zeigt die Tabelle noch einmal die grundlegenden Trends des Zeitalters permanenter Austerität: In den meisten Ländern blieben die Staatseinnahmen weitgehend konstant und auch die Staatsausgaben veränderten sich kaum. Von diesem Resultat weichen die sechs Überschussländer jedoch in beiden Dimensionen sichtbar ab: Zum einen sind sie die einzige Ländergruppe, die ihre Staatseinnahmen über den Beobachtungszeitraum konsistent reduzierte, zum anderen hielten sie ihre Staatsausgaben nicht nur konstant, sondern führten sie sogar deutlich zurück. Folgerichtig lagen die Staatseinnahmen der sechs Überschussländer im Jahr 2007 im Durchschnitt nur noch 3,2 Prozentpunkte über dem OECD-Mittel, nachdem dieser Wert im Jahr 1990 noch fast doppelt so hoch war. Und bei den Staatsausgaben reduzierte sich die Lücke sogar von 4,9 auf 0,9 Prozentpunkte.

Bereits die Entstehung der Überschüsse, die weitgehend im Zeitraum von 1990-1998 erfolgte, deutet den Zusammenhang von Überschüssen und Staatsrückbau an. Vor dem Hintergrund der Ergebnisse der Literatur zu Haushaltskonsolidierungen, der zufolge ausgabenseitige Konsolidierungen nachhaltiger sind als einnahmeseitige Konsolidierungen (Alesina u. Ardagna 2012), ist dieses Resultat wenig überraschend. Wird die Rolle der Bankenkrisen in Finnland und mit Abstrichen Dänemark berücksichtigt (die finnischen Staatsausgaben lagen 1994 zyklisch bereinigt bei 56,8 Prozent, in Dänemark bei 59,3 Prozent des BIP), dann

7 Eine Untersuchung von länderindividuelle Konsolidierungs- und Überschussperioden kommt zu sehr ähnlichen Ergebnissen. Der hier verwendete diachrone Vergleich macht es einfacher, die wesentlichen Aspekte in einer Tabelle zu erfassen. 


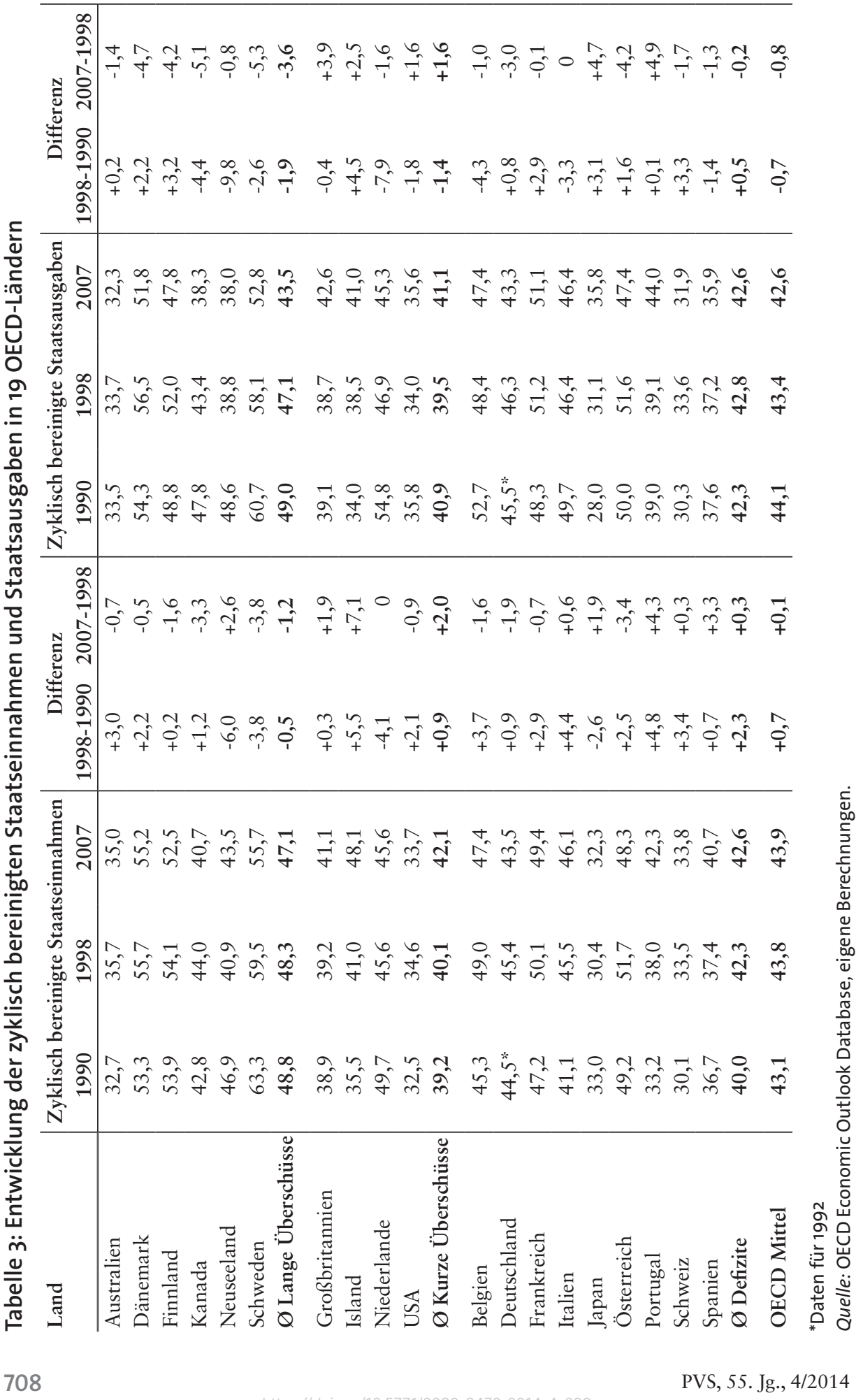


entstand der Überschuss mit der Ausnahme Australiens in allen Ländern weit überwiegend durch Kürzungen der Staatsausgaben. Diesen Befund der zyklisch bereinigten Zahlen bestätigen auch die auf der Kodierung politischer Entscheidungen basierenden Zahlen von DeVries et al. (2011) für Australien, Finnland, Kanada und Schweden. Neuseeland ist nicht Teil ihres Datensatzes und in Dänemark identifizieren sie zwar eine massive ausgabenseitige Konsolidierung zwischen 1983 und 1985, in den 1990er-Jahren aber überhaupt nur 1995 eine zudem sehr kleine Konsolidierung.

Die Konsolidierungsmaßnahmen und die ihnen in der Regel vorausgehende staatliche Schuldenkrise sind hier nur insofern von Bedeutung, als sie dazu beitrugen, dass sich in den untersuchten Ländern ein politisches Bekenntnis zu einer dauerhaft restriktiven Fiskalpolitik und einer strikten Haushaltsausgleichsnorm durchsetzen konnte. Im Zuge der Fiskalkrise wurde die Option defizitfinanzierter Politik grundlegend diskreditiert, und es wurden institutionelle Reformen ausgelöst, die die Möglichkeiten für eine solche Politik weitgehend beschränkten.

Die Auswirkungen der strikten Haushaltsausgleichsnorm zeigten sich aber vor allem in den Jahren nach 1998. Die Überschussländer hätten nach Überwindung der Fiskalkrise mit einem Wiederausbau der Staatstätigkeit beginnen können. Genau dies war die Befürchtung der Befürworter von „Starving the Beast“, die davor warnten, Überschüsse könnten den Anlass für neue Staatsausgaben bieten (Becker 2001; Friedman 2003). Empirisch war jedoch das Gegenteil der Fall: Die Staatsausgaben begannen nicht etwa zu steigen, sie gingen sogar weiter zurück. Das gilt auch nach Abzug der Zinsausgaben, die sich angesichts der fallenden Schuldenquote fast automatisch reduzierten. Die durch den Überschuss entstandenen fiskalischen Spielräume kamen kaum den Staatsausgaben zugute, sondern dienten dem Schuldenabbau und der Senkung der Staatseinnahmen. ${ }^{8}$

Wegen der geringen Fallzahl und des rein deskriptiven Charakters der Analyse sollten diese Befunde nicht überbewertet werden. Sie können nur einen ersten Eindruck von den empirischen Zusammenhängen bieten. ${ }^{9}$ Zudem können sie zwar zeigen, dass ein Rückbau der Staatstätigkeit stattfand, belegen aber noch nicht, dass dieser tatschlich mit einem strategisch verwendeten Bekenntnis zu dauerhaften Haushaltsüberschüssen zusammenhing. Dies kann sehr viel besser durch eine detaillierte Analyse einzelner Länder geleistet werden, die sich an diesen quantitativen Überblick anschließt.

Dabei erfolgt die Fallauswahl vor dem Hintergrund der Tatsache, dass es zwei mögliche Entwicklungspfade dauerhafter Haushaltsüberschüsse gibt, die auch die strategische Positionierung im Trilemma beeinflussen. Konservative Parteien können Überschüsse entweder von progressiven Vorgängerregierungen erben oder selbst durch Konsolidierungsmaßnahmen herbeiführen. Beide Entwicklungspfade sind Gegenstand der folgenden Fallstudien. Als Beispiel der ersten Konstellation

8 Dabei unterschätzen diese Zahlen vermutlich den zyklischen Charakter eines Teils der Staatseinnahmen in den rohstoffreichen Ökonomien Australiens, Kanadas und Neuseelands. Der tatsächliche Rückgang der Staatseinnahmen fiele demnach noch höher aus (Girouard u. André 2005; Turner 2006).

9 Eine sehr viel genauere Analyse der Entwicklung der Staatstätigkeit in den Überschussländern bieten Haffert u. Mehrtens (im Erscheinen). 
untersuchen wir Schweden, wo die bürgerliche Koalition im Jahr 2006 einen bereits seit acht Jahren bestehenden Überschuss von ihrer sozialdemokratischen Vorgängerregierung übernahm. Die zweite Konstellation repräsentiert Australien, wo eine seit 1996 regierende konservative Regierung den Überschuss herbeiführte, ehe sie 2007 von einer Labor-Regierung abgelöst wurde. Zwar hat Australien ein Zweiparteiensystem, während Schweden ein Mehrparteiensystem hat. In beiden Ländern handelt es sich aber um Block-Parteiensysteme, in denen ein linkes und ein rechtes Lager miteinander um die Regierungsmacht konkurrieren (GreenPedersen 2002).

\section{4. Überschüsse im „Volksheim“: Die fiskalische Strategie der „neuen Konservativen" in Schweden}

Vor dem Hintergrund der schwedischen politischen Geschichte und der positiven Einstellung der Bevölkerung zu Besteuerung und Sozialstaat befinden sich Befürworter eines schlanken Staates und von Steuersenkungen in einer schwierigen Ausgangslage. Eine fiskalische Strategie, die auf radikale Kürzungen der Sozialausgaben und eine umfassende Reduzierung der Steuerlast abzielt, ist nur äußerst schwer durchzusetzen. Die Verwirklichung des konservativen Ideals sinkender Steuern und Ausgaben in Verbindung mit einem ausgeglichenen Haushalt (rechte untere Ecke im Dreieck des Trilemmas der Fiskalpolitik) scheint in Schweden auf den ersten Blick unmöglich. Diese Fallstudie zeigt, wie es den Konservativen seit 2006 mit der „Overfeeding the Beast“-Strategie dennoch gelang, ihre fiskalpolitischen Ziele zu erreichen, die Staatsquote zu senken und im „sozialdemokratischen " Schweden 2010 wiedergewählt zu werden.

Schweden erlebte zu Beginn der 1990er-Jahre eine umfassende Wirtschafts-, Banken- und Schuldenkrise. Auf dem Höhepunkt dieser Krise waren die öffentlichen Haushaltsdefizite zweistellig und die Staatsverschuldung verdoppelte sich in kürzester Zeit fast von ca. 45 Prozent des BIP 1990 auf mehr als 80 Prozent 1994. Beide ideologischen Lager der schwedischen Politik begegneten der Staatsschuldenkrise mit einem strikten Konsolidierungsprogramm, durch das es letztlich gelang, die Krise unter Kontrolle zu bringen (Henriksson 2007).

Bei Ausbruch der Fiskalkrise wurde Schweden von einer konservativen Parteienkoalition regiert. Ein Großteil der Sparpolitik wurde jedoch bereits mit parlamentarischer Unterstützung der oppositionellen Sozialdemokraten verabschiedet, die 1994 an die Regierung zurückkehrten und noch umfangreichere Ausgabenkürzungen durchsetzten (Green 1995; Wenzelburger 2010). Gegen Ende der 1990er Jahre war die Staatsschuldenkrise endgültig überwunden, und die sozialdemokratischen Regierungen erzielten bis zum Regierungswechsel 2006 fast ausschließlich Budgetüberschüsse.

Trotz der grundlegenden Krise des Wirtschafts- und Sozialsystems und der einschneidenden Sparpolitik blieben die steuer- und sozialpolitischen Präferenzen der Bevölkerung weitgehend unverändert (Rothstein 1998). Der öffentliche Sektor und der generöse universelle Wohlfahrtsstaat sind in der schwedischen Wählerschaft weiterhin äußerst populär, die Zustimmung hat in den letzten Jahren sogar 
zugenommen und die Bereitschaft, sie mit entsprechend hohen Steuern und Abgaben zu finanzieren, ist ungebrochen (Svallfors 2011). Trotz erheblicher Kürzungen ist die schwedische Staatsquote im internationalen Vergleich weiterhin hoch.

Die hohe Popularität des Sozialstaats beschränkt zuvorderst die Strategiefähigkeit und die Wahlchancen der säkular-konservativen „Moderaten Sammlungspartei“ (Moderata samlingspartiet). Sie ist im traditionellen Fünfparteiensystem ${ }^{10}$ Schwedens ganz rechts außen angesiedelt und widersetzte sich stets am stärksten sozialdemokratischen Ideen von „Volksheim“, hohen Steuersätzen, Umverteilung und einem großen öffentlichen Sektor und positionierte sich als klare politische Alternative zu ihnen (Hancock 2002, S. 372). Diese politisch-ideologische Ausrichtung der Konservativen spiegelte sich bis vor wenigen Jahren auch in ihren Wahlprogrammen wider und führte zu wiederholten Wahlniederlagen. Alle früheren Versuche der konservativen Partei, den großen öffentlichen Sektor, die staatlichen Sozialausgaben und das Steuervolumen zu verkleinern, sind gescheitert. Wer das schwedische Steuer- und Sozialmodell offensiv attackierte, wurde entweder gar nicht erst in die Regierung gewählt oder verlor die Macht kurz danach wieder. In der Nachkriegszeit gelang es bürgerlichen Koalitionen bis 2006 nur zwei Mal, die Sozialdemokraten abzulösen $(1976,1991)$, und kurz darauf wurden sie wieder abgewählt (1982, 1994).

Hinzu kommt, dass die Höhepunkte von zwei schweren Staatsschuldenkrisen mit großen sozioökonomischen Verwerfungen jeweils in die Regierungszeit der Konservativen fielen. Obwohl die bürgerlichen Regierungen nicht die alleinige Verantwortung für den desolaten Zustand der öffentlichen Finanzen trugen, da sich die ökonomischen und finanziellen Probleme bereits über längere Zeitspannen aufgebaut hatten, setzte sich im kollektiven schwedischen Gedächtnis der Eindruck fest, das konservative Lager könne keine solide Finanzpolitik betreiben. Zweimal war es seine Aufgabe, eine große Finanz- und Wirtschaftskrise zu lösen, zweimal scheiterte es und hinterließ hohe Defizite.

Die fiskalische Strategie der Konservativen basierte bis 2006 im Prinzip auf der Reaganschen „Starving the Beast"-Logik, und die bürgerlichen Regierungen der Jahre 1976-1982 werden von Persson und Svensson (1989, S. 339) explizit als Beispiel für strategische Verschuldung angeführt: Sie nahmen hohe staatliche Defizite zur Erreichung ihres wichtigsten politisch-ideologischen Ziels, der Senkung von Steuern, in Kauf, ohne die Staatsausgaben in entsprechendem Umfang zu kürzen. Auch die Mitte-rechts-Regierung von Carl Bildt plante nach ihrem Wahlsieg 1991 trotz der sich abzeichnenden Schuldenkrise weitreichende Steuerentlastungen (Rexed 1999). Sie konnte diese letztlich aufgrund des Ausmaßes der Krise nur teilweise umsetzen. Trotzdem sanken zu Beginn der 1990er-Jahre unter einer konservativen Regierung die Staatseinnahmen, während die Staatsausgaben weiter anstiegen (Steinmo 2010, S. 65).

Mit dieser fiskalpolitischen Ausrichtung, die Steuern unter Inkaufnahme von Defiziten zu senken, sind die Konservativen wiederholt gescheitert und konnten

10 Mittlerweile hat sich in Schweden ein Achtparteiensystem herausgebildet und die rechtspopulistischen Schwedendemokraten bilden den rechten Rand des im Parlament vertretenen Parteienspektrums. 
keine erfolgreiche Alternative zu den Sozialdemokraten etablieren. Insbesondere bei der Reichstagswahl 2002 erlebte die konservative Partei, die mit einem besonders radikalen Steuersenkungs- und Liberalisierungsprogramm angetreten war, ein Wahldebakel, als sie mit ihrem Spitzenkandidaten Bo Lundgren nur knapp 15 Prozent der Stimmen erhielt.

In Folge dieser Wahlniederlage stellte sich die Partei unter dem Vorsitz von Fredrik Reinfeldt neu auf und orientierte sich deutlich zur politischen Mitte, was durch einen neuen Namenszusatz unterstrichen werden sollte. Ähnlich wie „New Labour" in Großbritannien, bezeichnen sich die Konservativen, seit Reinfeldt 2003 Parteichef wurde, als „Neue Moderate“ (Nya moderaterna). Ihre fiskalische Strategie beinhaltet als zentrales Element die Erzielung von ausgeglichenen Haushalten und kombiniert diese mit moderaten Ausgabenkürzungen und mittlerweile umfangreichen Steuersenkungen. Durch diese neue Prioritätensetzung, bei der die Erzielung eines Überschusses zunächst wichtiger als Steuersenkungen ist, wurde es möglich, beide Ziele langfristig umso besser zu verwirklichen und die schwedische Fiskalpolitik dauerhaft in Richtung der unteren rechten Ecke des Trilemmas zu verschieben.

Die beiden Protagonisten der unterschiedlichen fiskalischen Strategien der Konservativen, die Parteivorsitzenden Lundgren und Reinfeldt, äußern sich sehr ähnlich zu den Beweggründen für die strategische Neuausrichtung der Partei und betonen die Bedeutung einer soliden Haushaltspolitik. Der „alte“ Konservative Lundgren beurteilte die Situation nach der Wahlniederlage im Interview ${ }^{11}$ wie folgt:

And then they [the New Moderates Reinfeldt and Borg] started to ask themselves [...]: Why do we lose? One thing might be that we should focus on some questions that are easy to handle. To focus is one thing. And still a lot of non-socialist people and voters said: why did you let the deficit increase that much? They did not trust us with the public finances. [...]. The main reason from the beginning to focus that much on the debt reduction was to get rid of the perception that a non-socialist government is not good at public finance.

Lundgrens Erklärung wird von Reinfeldt bestätigt: „[T] he doubts of many voters that Moderaternas proposals for tax cuts were compatible with a sound state of the public finances was one reason that the party lost voters in the election in 2002“ (zitiert nach Lindbom 2006, S. 16). Die neue Parteiführung kam zu der Schlussfolgerung, dass die scharfen Attacken auf den Wohlfahrtsstaat und die permanenten Forderungen nach Steuersenkungen in Kombination mit der kläglichen haushaltspolitischen Bilanz der Partei die Hauptgründe für die schlechten Wahlergebnisse waren.

Die fiskalische Strategie, den Schwerpunkt der Fiskalpolitik auf die Erzielung von Überschüssen und nicht auf Steuersenkungen zu legen, war bei den Reichstagswahlen 2006 und 2010 erfolgreich. Während die Sozialdemokraten die schlechtesten Wahlergebnisse seit den 1920er-Jahren erzielten, lagen die „neuen“ Konservativen bei den Stimmanteilen mit ihnen erstmals gleichauf und konnten

11 Interview mit Bo Lundgren am 29. Juni 2011 in Stockholm. 
2006 eine bürgerliche Regierungskoalition bilden, die 2010 bestätigt wurde. Mit ihrer neuen fiskalpolitischen Ausrichtung verfolgten die Konservativen eine Strategie der Entpolitisierung sozial- und steuerpolitischer Fragen, die auch in anderen Ländern mit großen universellen Wohlfahrtsstaaten zu Wahlerfolgen sozialstaatskritischer Parteien führte. Die Analyse der Wahlprogramme und der Wahlergebnisse in skandinavischen Ländern zeigt, dass konservative Parteien dort aufgrund der großen Bedeutung des Sozialstaats immer dann besonders erfolgreich sind, wenn sie sich als gemäßigte Kräfte in der politischen Mitte positionieren und wenn es ihnen gelingt, mit der Sozialpolitik das zentrale Politikfeld der linken Parteien zu besetzen, also „issue ownership“ über das Thema zu erlangen (Arndt 2014).

Das schwedische Beispiel zeigt, dass die fiskalische Strategie, der Erzielung eines Haushaltsüberschusses oberste Priorität einzuräumen, unter den Bedingungen eines sozialdemokratisch geprägten Wirtschafts- und Gesellschaftssystems mit hohen Staatsausgaben und Steuersätzen den Schlüssel zur Verwirklichung konservativer Politikziele bilden kann. Die Überschusspolitik beinhaltet dabei den strategischen Einsatz des Budgetsaldos zur Erreichung anderer Zwecke. Die grundlegenden Ziele der konservativen Partei haben sich in Schweden trotz des neuen Namenszusatzes nicht verändert. Weiterhin präferiert sie eine möglichst weitreichende Entlastung der Steuerzahler und die Zurückdrängung des Staates aus vielen Politikfeldern, wie eine Vergleichsstudie der konservativen Parteiprogramme bestätigt: „In order to win elections, the bourgeois parties are therefore expected to try to attract the support of the median voter, i. e. to adapt to welfare state popularity [...]. This adaptation is however only strategic, their real preferences remain the same" (Lindbom 2006, S. 6). Demnach beschränkt sich die sozialpolitische Neuorientierung der konservativen Partei vor allem auf die Rhetorik, während die konkreten politischen Maßnahmen in die entgegengesetzte Richtung weisen.

Die fiskalische Überschussstrategie erlaubte den Konservativen den öffentlichen Sektor in einem Umfang zurückzudrängen, zu liberalisieren und zu privatisieren, der in Schweden lange nicht für möglich gehalten wurde (Blomqvist 2004). Indem die Regierung öffentliche Angebote unterfinanziert und parallel private Alternativen bei Kinderbetreuung, Altenpflege oder Schulen durch Subventionen und das Steuersystem fördert, ändert sie die Anreizstrukturen in den sozialen Sicherungssystemen. Dabei entfaltet die Kombination von Überschusspolitik und graduellem institutionellen Wandel durch „Layering“ eine transformative Wirkung, die das Potenzial besitzt, den schwedischen Wohlfahrtsstaat nachhaltig in Richtung eines liberalen Modells der sozialen Sicherung zu verändern (Mehrtens 2014).

Die schwedische Staatsquote fällt seit zwei Dekaden kontinuierlich und wurde allein in den letzten 10 Jahren um ca. 10 Prozentpunkte des BIP gesenkt (OECD 2013). Obwohl der schwedische Staat mehr einnimmt als er ausgibt, findet ein stetiger Rückbau staatlicher Aktivitäten und nicht etwa staatliche Expansion statt. Allein die offene Ankündigung eines derart umfangreichen Staatsabbaus und institutionellen Wandels im Steuer- und Sozialsystem wurde in der Vergangenheit von der schwedischen Wählerschaft abgestraft. Mittlerweile wird er in Kombination mit einer soliden Haushaltsführung umgesetzt. Seit der Steuerre- 
form 1990/91 wurden automatische Einnahmesteigerungen durch die „kalte Progression" abgeschafft, und durch diverse Entlastungen der oberen Einkommensgruppen in den 2000er-Jahren wurde das Steuersystem regressiver (Finanzpolitischer Rat 2008).

Gleichzeitig gibt es in wichtigen sozialpolitischen Ausgabenfeldern automatische Kostendeckelungen. Seit Inkrafttreten der Rentenreform 1994/98 wird das Rentenniveau automatisch der Einnahmesituation im Rentensystem angepasst, was vor dem Hintergrund der demografischen Entwicklung zu Rentenkürzungen führt (Settergren 2001). Ähnliche Auswirkungen hat die Reform der Arbeitslosenversicherung, deren Lohnersatzrate aufgrund einer fixen absoluten Obergrenze mittlerweile selten die eigentlich garantierten 80 Prozent erreicht und für einen durchschnittlichen Arbeitnehmer nur noch 50 Prozent beträgt (Davidsson u. Marx 2013). Diese Reformen bewirken, dass Staatsausgaben ohne aktives politisches Eingreifen automatisch sinken, während für eine Ausweitung der Staatstätigkeit politische Mehrheiten organisiert werden müssen.

Aufgrund der wechselhaften fiskalpolitischen Vergangenheit des Landes mit zwei gravierenden Verschuldungskrisen, die noch immer im kollektiven Gedächtnis verankert sind, herrscht in Schweden ein politisches Klima, das sehr sensibel auf öffentliche Defizite reagiert. Ausgeglichene Haushalte haben deshalb eine hohe symbolische Bedeutung erlangt. Die Erzielung eines strukturellen Überschusses wurde von der Regierung selbst in der tiefsten Rezession seit dem Zweiten Weltkrieg im Jahr 2009 nicht infrage gestellt und hatte entgegen dem Rat von fiskalpolitischen Experten gegenüber umfassenden konjunkturellen Stimuli Priorität (Finanzpolitischer Rat 2009).

Momentan gelingt es den Konservativen, ihre präferierte fiskalpolitische Konstellation im Trilemma der Fiskalpolitik zu verwirklichen und es gibt keine Anzeichen, dass sie in naher Zukunft davon abrücken müssten. Die Sozialdemokraten sind gezwungen, ihre fiskalpolitische Strategie an die der Konservativen anzupassen. Sie fordern ebenfalls ausgeglichene Haushalte und versprechen die von der aktuellen Regierung durchgesetzten Steuersenkungen auch nach einem Regierungswechsel nicht zu revidieren.

Stattdessen versuchen sie sogar, die Konservativen bei den Sparvorgaben noch zu überbieten. Die sozialdemokratische Schattenfinanzministerin Magdalena Andersson forderte beim Haushaltsentwurf 2012 mehr Sparsamkeit ein und veranschlagte in ihrem Budget fünf Mrd. Kronen weniger als die Regierung (Gmeiner 2013, S. 12). Da die Sozialdemokraten viele der Konsolidierungsmaßnahmen in der Vergangenheit zur Überwindung der Schuldenkrise und der Herbeiführung der Überschüsse selbst beschlossen, bestehen für sie kaum Möglichkeiten der offenen Kritik an der Sparpolitik der Regierung, da sie sich sonst unglaubwürdig machen würden und ihre eigene Politik aus der Vergangenheit heute als falsch darstellen müssten. Ein Wechsel der fiskalischen Strategie zurück zu höheren Staatsausgaben unter Inkaufnahme von Defiziten oder die Steigerung der Staatseinnahmen erscheint deshalb auch nach der Wahl im September 2014 unwahrscheinlich (Haffert u. Mehrtens im Erscheinen). 


\section{5. Überschüsse als Inbegriff fiskalpolitischen Verantwortungsbewusstseins: Australien}

Verglichen mit Schweden sind die Voraussetzungen für die Verwirklichung konservativer Politikziele in Australien wesentlich günstiger. So wird das Land in gängigen Typologien üblicherweise den liberalen Marktwirtschaften und den liberalen Wohlfahrtstaaten zugerechnet. Außerdem stellte die konservative Koalition aus Liberal Party und National Party zwischen 1949 und 1983 in 31 von 34 Jahren die Regierung. Diese Position als „natürliche Regierungspartei“ wurde jedoch durch fünf Wahlniederlagen in Folge infrage gestellt, als Labor von 1983 bis 1996 die Regierung bildete. Für die Liberalen besonders schmerzhaft war die Wahl des Jahres 1993, als sie mit einem radikalen ökonomischen Reformprogramm unter dem Titel „Fightback!“ antraten und eine bereits gewonnen geglaubte Wahl noch verloren. Auch in einem Land wie Australien war eine Politik des radikalen Staatsrückbaus also kaum erfolgreich durchzusetzen. ${ }^{12}$ Erst 1996 kamen die Konservativen unter John Howard wieder an die Regierung, nachdem sie kontroverse Positionen in der Wahlkampagne weitgehend vermieden.

Stattdessen nutzten sie vor allem die Schwächen der Labor-Regierung und attackierten diese für ökonomische Fehlentwicklungen, insbesondere das hohe Leistungsbilanzdefizit und die hohe Inflationsrate, auf die die australische Notenbank mit einer extrem restriktiven Geldpolitik reagierte (Brown 1991). In Verbindung mit Premierminister Keatings Fehler, die Rezession des Jahres 1990 als „the recession we had to have" (McFarlane 2006) herunterzuspielen, hinterließen die wachsende Auslandsverschuldung und die hohen Zinsen einen erheblichen Makel auf Labors wirtschaftspolitischer Reputation. Dieser verfestigte sich endgültig, als Keating in der Wahlkampagne des Jahres 1996 wider besseres Wissen bis zuletzt behauptete, das Budget sei ausgeglichen (Fenna 2007). Labor erbte also ein erhebliches Glaubwürdigkeitsproblem, das die Konservativen in den folgenden Jahren immer wieder erfolgreich thematisierten.

Die neue konservative Regierung legte gleich in ihrem ersten Budget die Grundlage für alle folgenden Überschüsse. Wenngleich die darin enthaltenen Sparanstrengungen weit hinter den Einschnitten in Schweden zurückblieben, handelte es sich um das restriktivste Budget der jüngeren australischen Geschichte. Finanzminister Costello kürzte die Staatsausgaben bis 1998 um insgesamt zwei Prozent des BIP (OECD AUS 1997, S. 58). Trotz dieser Kürzungen äußerten sich in einer Umfrage 59 Prozent der Befragten positiv über das Budget, der höchste Wert seit 1987 (Newspoll 1996).

Diese Politik führte von 1998 an zu Haushaltsüberschüssen, die jedoch rasch von der Asienkrise und den Kosten für den Militäreinsatz in Osttimor infrage gestellt wurden. Die Reaktion auf diese beiden Herausforderungen zeigte, welche Priorität die Regierung dem Bekenntnis zu Überschüssen einräumte. So leitete sie angesichts der von der Asienkrise ausgelösten Verwerfungen eine neue Runde von Ausgabenkürzungen ein, um das Budget im Überschuss zu halten (Costello 2008, S. 174). Als Australien im Jahr 1999 die Führung der UN-Mission INTERFET

12 Siehe dazu auch Martin (2011). 
auf Osttimor übernahm, kündigte Costello zudem die Einführung einer „Timor Tax“ an, um die Kosten des Einsatzes zu finanzieren. Letztlich wurde diese Sondersteuer nach längeren politischen Auseinandersetzungen doch nicht eingeführt, weil deutlich wurde, dass das Budget auch ohne sie im Überschuss bleiben würde (Costello 2000). Die Debatte um diese Steuer zeigt aber, dass die Konservativen im Zweifelsfall bereit waren, das Ziel niedriger Steuern hinter das Bekenntnis zu fortgesetzten Überschüssen zurückzustellen.

Zugleich gelang es der Regierung, auch die Labor-Opposition auf dieses Ziel zu verpflichten und somit eine allseits geteilte Haushaltsausgleichsnorm zu etablieren. Diese wurde durch die Einführung einer „Charter of Budget Honesty“ (CBH) im Jahr 1997 auch institutionell verankert. Neben umfangreichen Transparenzbestimmungen enthält sie eine Verpflichtung der Regierung zu einem über den Konjunkturzyklus ausgeglichenen Budget (Gruen u. Sayegh 2005, S. 631). Um fiskalpolitische Disziplin in Wahlkämpfen zu garantieren, verpflichtet sie Regierung wie Opposition zudem, ihre Wahlversprechen einer finanziellen Bewertung durch das Finanzministerium zu unterziehen. Die Verabschiedung der CBH folgte aber nicht allein fiskalischen, sondern auch parteipolitischen Erwägungen. Sie erlaubte den Konservativen, sich von der „fiskalischen Verantwortungslosigkeit“ ihrer Vorgänger abzusetzen und diese zugleich als politisches Argument lebendig zu halten. So war bereits ihr Titel ein geschicktes rhetorisches Spiel mit der „dishonesty“ der Regierung Keating im Wahlkampf 1996 (Wanna 2006) und Costello nutzte die Parlamentsdebatte über das Gesetz, um Labor hart zu attackieren:

This government has been repairing the fiscal mess left by the Labor Party. Before we came to office, we were told the budget was in surplus. It was not; it was in deficit to the tune of $\$ 10$ billion. [...] This bill will help to ensure that the fiscal profligacy of our predecessors is not repeated (Costello 1997).

Ab 2003 profitierte Australien von einem starken Rohstoffboom. Dieser war aber nicht der Grund für die fortgesetzten Überschüsse, die bereits seit einigen Jahren fest etabliert waren. Er warf jedoch die Frage auf, was mit den zusätzlichen Staatseinnahmen geschehen solle. Finanzminister Costello rief deshalb im Jahr 2004 unter dem wohlklingenden Namen „Future Fund“ einen Fonds ins Leben, der die Überschüsse für zukünftige Pensionsverpflichtungen ansparen und bis zum Jahr 2020 insgesamt 140 Mrd. Dollar ansammeln sollte (Gruen u. Sayegh 2005, S. 633). Politisch war dieser Fonds in vielerlei Hinsicht attraktiv: Nicht nur stärkte er die Reputation der konservativen Regierung für verantwortungsvolle Fiskalpolitik, er erzeugte auch die Notwendigkeit, in Zukunft weitere Überschüsse zu erzielen. Nicht zuletzt entzog er diese Überschüsse aber zugleich dem Zugriff der Politik und begegnete somit der konservativen Befürchtung, Überschüsse würden Anlass zu einer Erhöhung der Staatsausgaben bieten. So sah sich Labor heftiger Kritik ausgesetzt, als die Partei vorschlug, einen Teil des Fondsvermögens für Infrastrukturinvestitionen zu verwenden (Murphy 2007).

Neben der Einrichtung dieses Fonds erlaubten die Rohstoffeinnahmen aber vor allem, die Überschusspolitik mit umfangreichen Steuersenkungen zu verbinden und der von den Konservativen präferierten Lösung des Trilemmas damit sehr 
nahe zu kommen. Dabei wies Costello von Anfang an auf den engen Zusammenhang zwischen Überschüssen und Steuersenkungen hin: „Australian taxpayers are shareholders in Australia and they will benefit through income tax cuts as government debt is eliminated" (Gittins 1999).

Die Regierung hatte ihr Steuerreformprogramm bereits 1998 mit dem Beschluss über die Einführung einer "Goods and Services Tax“ begonnen und parallel dazu die Tarifbänder der Einkommensteuer deutlich verbreitert (OECD AUS 1999, S. 95). Nach einer Atempause zu Beginn des neuen Jahrtausends wurden immer größere Steuersenkungspakte dann zu einem Kernstück der konservativen Budgets. In jährlichen Steuersenkungsrunden leitete die Regierung somit große Teile der Rohstoffeinnahmen an die Steuerzahler weiter. Dabei verfolgte die Regierung explizit das Ziel, das Budget im Überschuss zu halten, ein Anwachsen des Überschusses auf mehr als ein Prozent des BIP aber zu verhindern (Costello 2008, S. 278). In Verbindung mit dem Future Fund stellte dies sicher, dass keine freien Ressourcen für politische Projekte der Opposition verfügbar waren. Wollte diese neue Politikprogramme vorschlagen, konnte sie zu deren Finanzierung nicht auf den Überschuss verweisen, sondern musste entweder andere Ausgaben streichen oder Steuererhöhungen ankündigen.

Diese politische Strategie war möglich, weil Labor sich mindestens ebenso sehr zu einer Fortsetzung der Überschusspolitik bekannte, wie die Konservativen selbst. Es gab daher praktisch keine politischen Konflikte um die Überschusspolitik. Vor der Wahl des Jahres 1998 hatte Labor zeitweilig eine klassische linke Position erwogen, musste jedoch bald die Popularität der Überschüsse erkennen und schwenkte auf die konservative Position ein: „By the time the 2001 election was drawing near, Labor became locked into the quite fanciful notion of the imperative of surplus budgets no matter what the stage of Australia's budget cycle“ (Battin 2002, S. 3).

Labor verfolgte nun eine Strategie der Differenzminimierung gegenüber den Konservativen (Battin 2002, S. 1). Ein Grund dafür war, dass die konservative Wirtschaftspolitik in vieler Hinsicht in der liberalisierenden Tradition der Laborregierungen der 1980er- und 1990er-Jahre stand. Zudem hatten die Konservativen Labor in den Wahlkämpfen von 1998, 2001 und 2004 von der Regierung mit Verweis auf die erste Hälfte der 1990er-Jahre immer wieder erfolgreich als die Partei von Haushaltsdefiziten, Inflation und hohen Zinsen attackiert. In ihrem Versuch, fiskalisches Verantwortungsbewusstsein zu beweisen, zeigte die LaborFührung sogar Ansätze einer konservativen „supply-side“-Rhetorik. So kritisierte Oppositionsführer Simon Crean 2003, das konservative Budget biete „the smallest tax cut from the highest taxing government in our history “ (Crean 2003).

Erst 2007 verloren die Konservativen schließlich die Wahl. Doch obgleich die Regierung wechselte, blieb der fiskalische Kurs fast unverändert. Die neugewählte Regierung bemühte sich von Anfang an, ihre fiskalpolitische Seriosität zu beweisen (Walter u. Uhr 2013, S. 437). Schon im Wahlkampf hatte der neue Premierminister Kevin Rudd „issue compensation“ (Jensen 2010) betrieben und sich als ökonomisch konservative Alternative zu Howard präsentiert (Williams 2008, S. 117). In seiner ersten Budgetrede erklärte Finanzminister Wayne Swan sogar: "The Government has made sure every single cent of new spending for the coming year has been more than met by savings elsewhere [...]“" (Swan 2008). 
Bald darauf wurde die Labor-Regierung jedoch mit den Auswirkungen der Weltfinanzkrise konfrontiert. Ihre Reaktion darauf blieb von einem klaren Bekenntnis zu Überschüssen geprägt. Zwar verabschiedete sie nach einigem Zögern eines der größten Konjunkturpakete in der gesamten OECD (OECD AUS 2010, S. 30, 36). Sie verband dieses deficit spending jedoch mit dem Versprechen, so schnell wie möglich in den Überschuss zurückzukehren. Das Ziel neuerlicher Überschüsse erlangte auch rasch eine enorme politische Bedeutung (Walter u. Uhr 2013). Die Regierung hatte zunächst angekündigt, das Budget im Haushaltsjahr 2015-2016 wieder auszugleichen. Dies erlaubte den Konservativen jedoch umgehend, dieses Ziel als unzureichend zu attackieren (Turnbull 2009).

Vor diesem Hintergrund zog Labor das Überschussziel im Vorfeld der Wahl des Jahres 2010 um drei Jahre auf das Haushaltsjahr 2012-2013 nach vorne. Obwohl sich dieses Ziel schon bald als unrealistisch erwies, sah sich die Regierung unter dem Druck, ihre Glaubwürdigkeit zu beweisen, und bekräftigte den Termin für neuerliche Überschüsse noch im Frühjahr 2012. Nur zwölf Monate später musste sie dann eingestehen, dass Überschüsse frühestens 2016 erreicht würden. Ohne den konservativen Wahlsieg im Herbst 2013 diesen Ereignissen zuschreiben zu wollen, boten sie der Opposition doch umfangreiche Gelegenheit für erfolgreiche politische Attacken (Walter u. Uhr 2013). ${ }^{13}$

Seit September 2013 hat Australien wieder eine konservative Regierung. Diese agiert unter den Bedingungen eines wohletablierten Allparteienkonsenses über die Notwendigkeit von Überschüssen. In der kurzen Frist werden die existierenden Defizite daher Anlass zu neuen Sparmaßnahmen geben. Auf längere Sicht wird aber auch eine Rückkehr zu Überschüssen die Verpflichtung der Regierung zu einer Politik fiskalpolitischer Zurückhaltung kaum infrage stellen. Inwiefern diese Politik bereits zu einem Rückbau des australischen Staates geführt hat, ist umstritten. Unter Verweis auf aggregierte Ausgabendaten betonen einige Beobachter, der australische Staat sei in den letzten zwei Jahrzehnten sogar gewachsen (so etwa Martin 2011). Dagegen kommen andere Autoren (so etwa Aulich 2010, siehe auch Errington u. Van Onselen 2007, S. 391) zu dem Schluss, faktisch habe sich die Rolle des Staates in der Wirtschaft deutlich reduziert. Tatsächlich senkte die konservative Regierung von John Howard die Staatsausgabenquote in ihrer Amtszeit um etwa drei Prozentpunkte (OECD 2013). Gemessen an den Einschnitten in Schweden ist dies zwar nur ein maßvoller Rückgang, aber vor dem Hintergrund der ständig wachsenden Rohstoffeinnahmen, die potenziell auch eine erhebliche Ausweitung der Staatstätigkeit hätten finanzieren können, zeigt er den Erfolg der konservativen Strategie.

13 Zu Labors allgemeinen Schwierigkeit, eine kohärente Fiskalpolitik „,in Howard's shadow “ zu formulieren, siehe auch Wilson (2013), der allerdings nicht genauer auf die Rolle des Überschussziels eingeht. 


\section{Fazit}

Ronald Reagan war nicht der einzige konservative Politiker, der erfahren musste, dass „big government“ ein Biest ist, das nur sehr schwer auszuhungern ist. Konservative Bemühungen, den Staat zurückzudrängen, trafen fast überall auf energischen Widerstand. Während es einigen Regierungen gelang, die Steuern zu senken, scheiterten sie regelmäßig daran, die nötigen Ausgabenkürzungen durchzusetzen, um die Steuersenkungen zu finanzieren. Weil „Starving the Beast” die Unterstützung einer politisch bindenden Haushaltsausgleichsnorm fehlte, führten die Steuersenkungen zu höheren Defiziten und nicht zu sinkenden Ausgaben.

In diesem Artikel haben wir Länder analysiert, in denen der Parteienwettbewerb nicht vor dem Hintergrund permanenter Defizite, sondern im Kontext permanenter Überschüsse stattfindet. Die Dynamik des Parteienwettbewerbs unter diesen Bedingungen wurde in den Fallstudien Schwedens und Australiens deutlich. Dabei war die grundlegende Dynamik in beiden Ländern trotz der sehr unterschiedlichen institutionellen Voraussetzungen sehr ähnlich. Das Bekenntnis zu Überschüssen war ein Instrument, mit dem konservative Parteien sich vor Kritik durch die politische Linke schützen und diese zugleich unter strategischen Zugzwang setzen konnten. Die Popularität ausgeglichener Haushalte und die hohe symbolische Aufladung der Haushaltsausgleichsnorm machte es dieser unmöglich, auch nur implizit für die Finanzierung wachsender Staatsausgaben durch Haushaltsdefizite (obere Ecke des Trilemmas) einzutreten. Es war ihr jedoch ebenfalls nicht möglich, sich hinter unkonkreten Prognosen zukünftiger Gegenfinanzierung zu verstecken, ohne sich dem Vorwurf fiskalpolitischer Verantwortungslosigkeit auszusetzen. Vor diesem Hintergrund vermieden die linken Parteien eine Politisierung der Überschusspolitik. Zugleich zwang sie dies an der Regierung jedoch dazu, eine Politik zu verfolgen, die sich kaum von der ihrer konservativen Vorgänger unterschied. Denn wenn sie den Überschuss verlor oder jedenfalls zu verlieren drohte, ermöglichte dies der Opposition, der Regierung eine Gefährdung hart errungener Konsolidierungserfolge vorzuwerfen. Das festigte die Überschusspolitik weiter. Aus unterschiedlichen strategischen Motiven konvergierten beide politischen Lager daher zu einer sehr ähnlichen Politik (Haffert u. Mehrtens 2014).

Von diesem Allparteienkonsens über die Fortsetzung der Überschusspolitik profitierten vor allem die Ziele konservativer Parteien. Denn das unbedingte Bekenntnis zu ausgeglichen Haushalten erlaubte ihnen, trotz wachsender fiskalischer Ressourcen einen permanenten Druck auf die Ausgabenseite des Budgets aufrechtzuerhalten. ${ }^{14}$ Während konservative Versuche, einen direkten Rückbau der Staatstätigkeit durchzusetzen, also fast überall auf entschlossenen Widerstand trafen, war ihnen ein wesentlich größerer Erfolg beschieden, wo sie sich explizit in den Mantel fiskalischen Verantwortungsbewusstseins hüllten.

Diese Strategie erlaubte eine graduelle, aber dennoch substanzielle Reduktion der Rolle des Staates in der Wirtschaft und zum Teil umfangreiche Steuersenkungen. Dabei erfolgte der Rückbau der Staatsausgaben kaum durch echte „Kürzungen“ im Wortsinne, also in nominalen Zahlen. Die „Overfeeding the Beast“-Stra-

14 Siehe dazu ausführlich Haffert u. Mehrtens im Erscheinen. 
tegie bestand vielmehr darin, das Wachstum der Staatsausgaben unter Verweis auf die Haushaltsausgleichsnorm zu bremsen und ansonsten auf die erodierende Kraft von Wirtschaftswachstum, Inflation und dem Vergehen der Zeit zu setzen. In den Worten der Theorie graduellen institutionellen Wandels handelt es sich also um Staatsrückbau durch „Drift“, nicht durch „Disruption“ (Streeck u. Thelen 2005). Auch wurde die Bevölkerung keineswegs von einer plötzlichen Begeisterung für Staatsabbau erfasst. Vielmehr blieben die Wohlfahrtsstaaten beider Länder enorm populär (Svallfors 2011; Martin 2011). Gleichzeitig fand das Ziel eines ausgeglichenen Haushalts aber breite öffentliche Unterstützung, selbst wenn es eine effektive Schranke für jeden Ausbau öffentlicher Leistungen bedeutete.

Bezogen auf das Trilemma-Modell erwies sich eine Betonung ausgeglichener Haushalte gegenüber einer Betonung niedriger Steuern also als die erfolgreichere Strategie. Die konservativen Parteien in Australien und Schweden konzentrierten sich zunächst auf den Budgetsaldo, um mithilfe der Überschüsse dann die zunächst aufgeschobenen Steuersenkungen zu finanzieren. Der entscheidende Vorteil dieser Strategie lag darin, dass progressive Parteien ihr kaum etwas entgegensetzen konnten. Gegen eine frontale Attacke auf den Wohlfahrtsstaat konnten sie opponieren und damit sogar Popularitätsgewinne erzielen. Die fortlaufenden Überschüsse zu kritisieren, wäre dagegen hochgradig unpopulär gewesen, auch wenn mit ihnen ein schleichender Staatsrückbau durch „Layering“ verbunden war. Indem die Konservativen die frei werdenden Ressourcen in Steuersenkungen umlenkten, erzielten sie den paradox wirkenden Erfolg, einen fiskalisch immer weniger unter Druck stehenden Staat auf permanentem Austeritätskurs zu halten.

\section{Literatur}

Adams, James, und Zeynep Somer-Topcu. 2009. Policy Adjustment by Parties in Response to Rival Parties' Policy Shifts: Spatial Theory and the Dynamics of Party Competition in Twenty-Five Post-War Democracies. British Journal of Political Science 39: 825-846. DOI: $10.1017 /$ S0007123409000635.

Alesina, Alberto, und Silvia Ardagna. 2012. The Design of Fiscal Adjustments. NBER Working Paper 18423. Cambridge, MA: National Bureau of Economic Research. DOI: $10.3386 / \mathrm{w} 18423$.

Alesina, Alberto, und Guido Tabellini. 1990. A Positive Theory of Fiscal Deficits and Government Debt. Review of Economic Studies 57: 403-414. DOI: 10.2307/2298021.

Arndt, Christoph. 2014. Beating Social Democracy on Its Own Turf: Issue Convergence as Winning Formula for the Centre-Right in Universal Welfare States. Scandinavian Political Studies 37: 149-170. DOI: 10.1111/1467-9477.12018.

Aulich, Chris. 2010. Reconstructing the Australian State: The Privatization Factor. In Dealing With Debt: Lessons From Abroad, Hrsg. John Springford, 74-92. London: Centre Forum.

Bartlett, Bruce. 2007. "Starve the Beast”. Origins and Development of a Budgetary Metaphor. The Independent Review 12: 5-26.

Battin, Tim. 2002. The Australian Labor Party and the Notion of Economic Management. Journal of Economic and Social Policy 6: 1-12.

Becker, Gary S. 2001. The Real Reason We Need a Tax Cut. Business Week 28, 19.03.2001. 
Blomqvist, Paula. 2004. The Choice Revolution: Privatization of Swedish Welfare Services in the 1990s. Social Policy and Administration 38: 139-155. DOI: 10.1111/j.14679515.2004.00382.x.

Boix, Carles. 2000. Partisan Governments, the International Economy, and Macroeconomic Policies in Advanced Nations, 1960-93. World Politics 53: 38-73. DOI: 10.1017/ S0043887100009370.

Brender, Adi, und Allan Drazen. 2008. How Do Budget Deficits and Economic Growth Affect Reelection Prospects? Evidence from a Large Panel of Countries. American Economic Review 98: 2203-2220. DOI: 10.1257/aer.98.5.2203.

Brown, Kevin. 1991. Riding to the End of a Wave. Financial Times, 24.05.1991.

Costello, Peter. 1997. Charter of Budget Honesty Bill 1996, Second Reading. Commonwealth of Australia, Parliamentary Debates, House of Representatives, 05.12.1997.

Costello, Peter. 2000. Budget Speech 2000-01. Department of the Treasury. Canberra.

Costello, Peter. 2008. The Costello Memoirs. Melbourne: Melbourne University Press.

Crean, Simon. 2003. Budget Debate. Commonwealth of Australia, Parliamentary Debates, House of Representatives, 15.03.2003.

Cusack, Thomas R. 1999. Partisan Politics and Fiscal Policy. Comparative Political Studies 32: 464-486. DOI: 10.1177/0010414099032004003.

Davidsson, Johan Bo, und Paul Marx. 2013. Losing the Issue, Losing the Vote: Issue Competition and the Reform of Unemployment Insurance in Germany and Sweden Political Studies 61: 505-522. DOI: 10.1111/j.1467-9248.2012.00997.x.

DeVries, Pete, Jaime Guajardo, Daniel Leigh, und Andrea Pescatori. 2011. A New Actionbased Dataset of Fiscal Consolidation. IMF Working Paper 11/128. Washington, D. C.: International Monetary Fund.

Errington, Wayne, und Peter Van Onselen. 2007. John Winston Howard. Melbourne: Melbourne University Press.

Fenna, Alan. 2007. Governing in Good Times: Fiscal Policy and Tax Reform in Australia 1996-2006. Australian Journal of Political Science 42: 329-350. DOI: 10.1080/ 10361140701320059.

Finanzpolitischer Rat 2008. Swedish Fiscal Policy. Report of the Swedish Fiscal Policy Council 2008. Stockholm: Finanspolitiska rådet.

Finanzpolitischer Rat 2009. Swedish Fiscal Policy. Report of the Swedish Fiscal Policy Council 2009. Stockholm: Finanspolitiska rådet.

Franzese, Robert J. 2002. Electoral and Partisan Cycles in Economic Policies and Outcomes. Annual Review of Political Science 5: 369-421. DOI: 10.1146/annurev.polisci.5.112801.080924.

Friedman, Milton. 2003. What Every American Wants. Wall Street Journal, 15.01.2003.

Girouard, Nathalie, und Christophe André. 2005. Measuring Cyclically-adjusted Budget Balances for OECD Countries. OECD Economics Department Working Paper 434. Paris: OECD. DOI: $10.1787 / 787626008442$.

Gittins, Ross. 1999. The high cost of paying less tax. Sydney Morning Herald, 25.08.1999.

Gmeiner, Jens. 2013. Die „Zukunftspartei“ Schwedens? Die Schwedische Sozialdemokratie zwischen erfolgreicher Vergangenheit und unsicherer Zukunft. Berlin: Friedrich-EbertStiftung.

Green-Pedersen, Christoffer. 2002. The Politics of Justification. Amsterdam: Amsterdam University Press. 
Green, John H. 1995. Public Finances, Adjustment Programs, and Debt Dynamics. In Challenges to the Swedish Welfare State, Hrsg. Desmond Lachman, Adam Bennett, John H. Green, Robert Hagemann und Ramana Ramaswamy, 20-29. Washington, D. C.: International Monetary Fund.

Gruen, David, und Amanda Sayegh. 2005. The Evolution of Fiscal Policy in Australia. Oxford Review of Economic Policy 21: 618-635. DOI: 10.1093/oxrep/gri035.

Haffert, Lukas. 2014. Freiheit von Schulden - Freiheit zur Gestaltung? Die politische Ökonomie von Haushaltsüberschüssen. Köln: Universität zu Köln.

Haffert, Lukas, und Philip Mehrtens. 2014. Haushaltsüberschüsse und ihre Verwendung: Wiedergewinnung staatlicher Handlungsfähigkeit? Zeitschrift für Staats- und Europawissenschaften (ZSE) 12: 210-241.

Haffert, Lukas, und Philip Mehrtens. Im Erscheinen. From Austerity to Expansion? Consolidation, Budget Surpluses, and the Decline of Fiscal Capacity. Politics \& Society.

Hancock, M. Donald. 2002. Sweden. In Politics in Europe. An Introduction to the Politics of the United Kingdom, France, Germany, Italy, Sweden, Russia, and the European Union, Hrsg. M. Donald Hancock, 347-401. Houndmills: Palgrave Macmillan.

Häusermann, Silja, Georg Picot, und Dominik Geering. 2013. Rethinking Party Politics and the Welfare State - Recent Advances in the Literature. British Journal of Political Science 43: 221-240. DOI: http://dx.doi.org/10.1017/S0007123412000336.

Hellwig, Timothy. 2012. Constructing Accountability: Party Position Taking and Economic Voting. Comparative Political Studies 45: 91-118. DOI: 10.1177/ 0010414011422516.

Henriksson, Jens. 2007. Ten Lessons About Budget Consolidation. Brüssel: Bruegel.

Hibbs, Douglas A., Jr. 1977. Political Parties and Macroeconomic Policy. American Political Science Review 71: 1467-1487. DOI: 10.1017/S0003055406232568.

Hicks, Alexander M., und Duane H. Swank. 1992. Politics, Institutions, and Welfare Spending in Industrialized Democracies, 1960-82. The American Political Science Review 86: 658-674. DOI: 10.2307/1964129.

Jensen, Carsten. 2010. Issue Compensation and Right-Wing Government Social Spending. European Journal of Political Research 49: 282-299. DOI: 10.1111/j.1475-6765. 2009.01898.x.

Kalyvas, Stathis N., und Kees Van Kersbergen. 2010. Christian Democracy. Annual Review of Political Science 13: 183-209. DOI: 10.1146/annurev.polisci.11.021406.172506.

Kitschelt, Herbert. 2001. Partisan Competition and Welfare State Retrenchment: When Do Politicians Choose Unpopular Policies? In The New Politics of the Welfare State, Hrsg. Paul Pierson, 265-302. Oxford: Oxford University Press.

Lindbom, Anders. 2006. The Swedish Conservative Party and the Welfare State: Institutional Change and Adapting Preferences. Arbetsrapport 2006: 12. Stockholm: Institutet för Framtidsstudier.

Martin, Aaron. 2011. Partisan Identification and Attitudes to Big Versus Small Government in Australia: Evidence from the ISSP. Australian Journal of Political Science 46: 243-256. DOI:10.1080/10361146.2011.567971.

McFarlane, Ian. 2006. The real reasons why it was the 1990s recession we had to have. The Age 02.12.2006. http://www.theage.com.au/news/business/the-real-reasons-why-it-wasthe-1990s-recession-we-had-to-have/2006/12/01/1164777791623.html. Zugegriffen: 24.10.2014. 
Mehrtens, Philip. 2014. Staatsschulden und Staatstätigkeit. Zur Transformation der politischen Ökonomie Schwedens. Frankfurt: Campus Verlag.

Morgan, Iwan W. 2009. The Age of Deficits. Presidents and Unbalanced Budgets from Jimmy Carter to George W. Bush. Lawrence: University Press of Kansas.

Murphy, Katharine. 2007. Labor seizes on Lib's Future Fund backing. The Age 27.03.2007. http://www.theage.com.au/news/national/labor-seizes-on-libs-future-fundbacking/2007/03/26/1174761379941.html. Zugegriffen: 24.10.2014.

Newspoll. 1996. Federal Budget: Impact on Economy. http://polling.newspoll.com.au.tmp. anchor.net.au/cgi-bin/polling/display_poll_data.pl. Zugegriffen: 24.10.2014.

Niskanen, William A. 2006. Limiting Government: The Failure of „Starve the Beast“. Cato Journal 26: 553-558.

OECD 2013. OECD Economic Outlook Database: Statistics and Projections. Paris: OECD.

OECD AUS. 1997. OECD Economic Survey: Australia. Paris: OECD.

OECD AUS. 1999. OECD Economic Survey: Australia. Paris: OECD.

OECD AUS. 2010. OECD Economic Survey: Australia. Paris: OECD.

Persson, Torsten, und Lars E. O. Svensson. 1989. Why a Stubborn Conservative would Run a Deficit: Policy with Time-Inconsistent Preferences. Quarterly Journal of Economics 104: 325-345. DOI: 10.2307/2937850.

Picot, Georg. 2009. Party Competition and Reforms of Unemployment Benefits in Germany: How a Small Change in Electoral Demand Can Make a Big Difference. German Politics 18: 155-179. DOI: 10.1080/09644000902874257.

Pierson, Paul. 2001. From Expansion to Austerity. The New Politics of Taxing and Spending. In Seeking the Center: Politics and Policymaking in the New Century, Hrsg. Martin A. Levin, Marc K. Landy und Martin Shapiro, 54-80. Washington, D. C.: Georgetown University Press.

Rexed, Knud. 1999. Schwedische Steuerpolitik. In Schweden im Wandel: Entwicklungen, Probleme, Perspektiven, Hrsg. Claudius H. Riegler und Olaf Schneider, 217-230. Berlin: BWV - Berliner Wissenschaftsverlag.

Romer, Christina, und David Romer. 2009. Do Tax Cuts Starve the Beast? The Effect of Tax Changes on Government Spending. NBER Working Paper No. 13548. Cambridge, MA: National Bureau of Economic Research.

Rothstein, Bo. 1998. Just Institutions Matter. The Moral and Political Logic of the Universal Welfare State. Cambridge: Cambridge University Press.

Settergren, Ole. 2001. The Automatic Balance Mechanism of the Swedish Pension System. Wirtschaftspolitische Blätter 48: 339-349.

Stein, Herbert. 1969. The Fiscal Revolution in America. Chicago: University of Chicago Press.

Steinmo, Sven. 2010. The Evolution of Modern States: Sweden, Japan, and the United States. Cambridge: Cambridge University Press.

Stockman, David. 1986. The Triumph of Politics. How the Reagan Revolution Failed. New York: Harper \& Row.

Streeck, Wolfgang, und Kathleen Thelen, 2005. Introduction: Institutional Change in Advanced Political Economies. In dies.: Beyond Continuity: Institutional Change in Advanced Political Economies, 3-39. Oxford: Oxford University Press. 
Svallfors, Stefan. 2011. A Bedrock of Support? Trends in Welfare State Attitudes in Sweden, 1981-2010. Social Policy \& Administration 45: 806-825. DOI: 10.1111/ j.1467-9515.2011.00796.x.

Swan, Wayne. 2008. Budget Speech 2008-09. Canberra: Department of the Treasury.

The New York Times. 25.01.1987. The State of the President.

Tufte, Edward R. 1978. Political Control of the Economy. Princeton: Princeton University Press.

Turnbull, Malcolm. 2009. Budget Debate. Commonwealth of Australia, Parliamentary Debates, House of Representatives, 14.05.2009.

Turner, David. 2006. Should Measures of Fiscal Stance be Adjusted for Terms of Trade Effects? OECD Economics Department Working Paper 519. Paris: OECD

Wagschal, Uwe. 1996. Staatsverschuldung: Ursachen im internationalen Vergleich. Opladen: Leske+Budrich.

Walter, Ryan, und John Uhr. 2013. Budget Talk: Rhetorical Constraints and Contests. Australian Journal of Political Science 48: 431-444. DOI: 10.1080/10361146.2013.837426.

Wanna, John. 2006. Between a Rock and a Hard Place: the Nonsense of Australia's Charter of Budget Honesty Act 1998. Paper presented at the Australasian Political Studies Association Conference. University of Newcastle.

Wenzelburger, Georg. 2010. Haushaltskonsolidierungen und Reformprozesse. Determinanten, Konsolidierungsprofile und Reformstrategien in der Analyse. Berlin: LIT Verlag.

Williams, Paul D. 2008. The 2007 Australian Federal Election: The Story of Labor's Return from the Electoral Wilderness. Australian Journal of Politics and History 54: 104125. DOI: $10.1111 /$ j.1467-8497.2008.00487.x.

Wilson, Shaun. 2013. The Limits of Low-Tax Social Democracy? Welfare, Tax and Fiscal Dilemmas for Labor in Government. Australian Journal of Political Science 48: 286306. DOI: 10.1080/10361146.2013.821102.

\section{Autorenangaben}

Lukas Haffert, M. A.

European University Institute, Max Weber Programme,

Via dei Roccettini 9,

I-50014 San Domenico di Fiesole,

lukas.haffert@eui.eu

Philip Mehrtens, Dr. rer. pol.

Max-Planck-Institut für Gesellschaftsforschung,

Paulstr. 3,

50676 Köln,

mehrtens@mpifg.de 